\title{
Diffraction Effects and Compensation in Passive Acoustic Mapping
}

\author{
Michael D. Gray ${ }^{\circledR}$, Erasmia Lyka, and Constantin C. Coussios
}

\begin{abstract}
Over the last decade, a variety of noninvasive techniques have been developed to monitor therapeutic ultrasound procedures in support of safety or efficacy assessments. One class of methods employs diagnostic ultrasound arrays to sense acoustic emissions, thereby providing a means to passively detect, localize, and quantify the strength of nonlinear sources, including cavitation. Real array element diffraction patterns may differ substantially from those presumed in existing beamforming algorithms. However, diffraction compensation has received limited treatment in passive and active imaging, and measured diffraction data have yet to be used for array response correction. The objectives of this paper were to identify differences between ideal and real element diffraction patterns, and to quantify the impact of diffraction correction on cavitation mapping beamformer performance. These objectives were addressed by performing calibration measurements on a diagnostic linear array, using the results to calculate diffraction correction terms, and applying the corrections to cavitation emission data collected from soft tissue phantom experiments. Measured diffraction patterns were found to differ significantly from those of ideal element forms, particularly at higher frequencies and shorter distances from the array. Diffraction compensation of array data resulted in cavitation energy estimates elevated by as much as a factor of 5 , accompanied by the elimination of a substantial bias between two established beamforming algorithms. These results illustrate the importance of using measured array responses to validate analytical field models and to minimize observation biases in imaging applications where quantitative analyses are critical for assessment of therapeutic safety and efficacy.
\end{abstract}

Index Terms-Array, cavitation, passive acoustic mapping (PAM), ultrasound.

\section{INTRODUCTION}

A GROWING number of therapeutic ultrasound applications require real-time monitoring for the purposes of cavitation activity optimization, utilization or avoidance [1]-[3]. Recently developed ultrasound-based passive [4]-[9] and active [10]-[12] cavitation mapping techniques offer the potential for improved treatment efficacy and safety by localizing and quantifying cavitation activity during therapeutic ultrasound procedures. While the details of these methods differ, those that are applied to tissues unobscured by bone predominantly operate on data collected with conventional diagnostic ultrasound arrays. In cavitation mapping simulations and experiments, individual array elements are

Manuscript received October 30, 2017; accepted November 24, 2017. Date of publication December 6, 2017; date of current version January 26, 2018. This work was supported by the National Institute for Health Research Oxford Biomedical Research Centre. (Corresponding author: Michael D. Gray.)

The authors are with the Department of Engineering Science, Institute of Biomedical Engineering, University of Oxford, Oxford OX3 7DQ, U.K. (e-mail: michael.gray@eng.ox.ac.uk).

Digital Object Identifier 10.1109/TUFFC.2017.2778509 typically treated as point receivers, so that their frequency response is independent of imaging target range and angle. However, the finite size of an element has a bandwidth-limiting effect [13]-[16] that varies by position in the beamformer field of view. Computed responses of finite-size array elements have been incorporated into B-mode ultrasound studies [17]-[19], as well as photoacoustic simulation [20], [21], calibration [22], and reconstruction [23], [24]. In some studies, computations were supported by close range pressure field measurements in order to quantify array lens effects [25], [26]. Notional far-field directivities have been used to compensate individual element data as a function of angle in the calculation of B-mode images [27], and numerical approximations of finite array element diffraction have been incorporated into a cavitation imaging beamformer [5]. However, the field pattern models used in the latter two studies were not directly validated against corresponding experiments. More generally, experimental methods for characterization and compensation of array element diffraction effects have yet to be applied to the development of passive cavitation mapping techniques.

Taken together, the above studies suggest that correction for real element response characteristics could increase useable signal bandwidth with commensurate improvements to energy estimation and spatial resolution. Some form of diffraction correction also may offer a potentially significant step toward elimination of system-dependent observation biases, since the impact of diffraction effects may vary with array geometry, beamforming apodization, imaging distance, and algorithm choice. Removal of such biases may enable absolute quantification of cavitation emissions. Just as a properly calibrated hydrophone reports pressure that may aid in the understanding of physical processes underlying the generated signal, a biasfree cavitation mapping methodology would provide more meaningful indications of safety and efficacy for bubbleinduced bioeffects, including blood-brain barrier opening [28], vascular damage [29], or thermal ablation [30].

Through simulation and experiment, this paper presents array element diffraction pattern examples, quantifies their impact on passively acquired maps of cavitation activity, and illustrates improvements obtained by diffraction pattern correction.

\section{Methods}

This section begins with a description of the passive acoustic mapping (PAM) algorithms employed, in order to specify the information required and assumptions made. This is followed by descriptions of the procedures used to characterize a commercially available diagnostic ultrasound array. Finally, 
an in vitro cavitation monitoring experiment is described, the data from which are used to quantify the impact of applying experimentally determined array diffraction corrections.

\section{A. Passive Mapping Algorithms}

In this paper, cavitation emissions received with a conventional ultrasound linear array were processed with two PAM algorithms adopted for cavitation monitoring: time exposure acoustics (TEA) [6] and robust Capon beamforming (RCB) [7]. The former provides a computationally simple method of mapping sources of cavitation at the expense of spatial resolution, while the latter provides superior spatial resolution but at considerable computational cost. These two algorithms are thought to bracket the resolution capabilities of known passive mapping algorithms published to date. Both algorithms operate on time-domain array response signals $p\left(t, \boldsymbol{r}_{j}\right)$ received at array element locations $\boldsymbol{r}_{j}$ for time duration $T$ to produce an estimate of monopolar source energy $E(\boldsymbol{r})$ at an observation location $r$

$$
E(\boldsymbol{r})=\frac{1}{4 \pi \rho c} \int_{t_{0}}^{t_{0}+T} q^{2}(\boldsymbol{r}, t) d t
$$

where $\rho$ and $c$ are the density and sound speed of the ambient medium, respectively, $t_{0}$ is the start time of the data record, and bold type quantities indicate a vector quantity. The source strength $q$ as a function of time $t$ is defined by a sum over the $j=1: N$ array elements

$$
q(\boldsymbol{r}, t)=\frac{1}{N} \sum_{j=1}^{N} 4 \pi w_{j}\left|\boldsymbol{r}_{j}-\boldsymbol{r}\right| p\left(\boldsymbol{r}_{j}, t+\left|\boldsymbol{r}_{j}-\boldsymbol{r}\right| / c\right)
$$

where $w_{j}$ are the weights applied to each element. All weights in the TEA beamformer are set to unity, while RCB optimizes the weights for each imaging location $\boldsymbol{r}$ in order to minimize the variance of the array output, with allowance for uncertainties in array element sensitivity and location [7], [31]. Here, the time window $t_{0} \leq t \leq\left(t_{0}+T\right)$ contains cavitation signals generated by pulsed ultrasound (Section II-D) of duration $\sim 0.8 T$, so that (1) is an estimate of the source energy associated with a finite sequence of events.

The delay and amplitude scaling operations with respect to the distance between element and imaging locations $\left(\left|\boldsymbol{r}_{j}-\boldsymbol{r}\right|\right)$ in (2) presume a point (omnidirectional) model for signal reception. In reality, both the finite array element size and the overlying lens may significantly modify the characteristics of the received signals. The predictions and measurements described in the next sections are used to quantify deviations from omnidirectionality, and form the basis of array element response correction prior to beamforming of cavitation data.

\section{B. Diffraction Pattern Prediction}

To establish an analytical baseline for comparison with measurements described in Section II-C, diffraction patterns for elements of width $L_{x}$ and height $L_{y}$ were calculated as a function of frequency $(f)$ and location using a simplified form of the Rayleigh integral

$$
D_{R, j}(f, x, z)=\frac{R_{j}}{S} \iint_{S} \frac{e^{-i k R_{s}}}{R_{S}} d S
$$

where the area of element $S=L_{x} L_{y}, R_{j}=\left|\boldsymbol{r}_{j}-\boldsymbol{r}\right|$ [see (2)], $R_{s}=\left(\left(x_{s}-x\right)^{2}+\left(y_{s}-y\right)^{2}+\left(z_{s}-z\right)^{2}\right)^{1 / 2}, x_{s}$ and $y_{s}$ are locations within the element area $\left(-(1 / 2) L_{x} \leq x_{s} \leq\right.$ $\left.(1 / 2) L_{x},-(1 / 2) L_{y} \leq y_{s} \leq(1 / 2) L_{y}\right), z_{s}=0, k=(2 \pi f / c)$, and the spatially invariant scaling terms related to velocity distribution and medium properties [32] were omitted. Elevation focusing effects were calculated by incorporating phase shifts along the element height

$$
D_{R j, \mathrm{EF}}(f, x, z)=\frac{R_{j}}{S} \iint_{S} \frac{e^{-i k\left(R_{s}-R_{\mathrm{EF}}\right)}}{R_{S}} d S
$$

where $R_{\mathrm{EF}}=\left(\left(y_{s}-y\right)^{2}+\left(z_{s}-z_{\mathrm{EF}}\right)^{2}\right)^{1 / 2}$ and $z_{\mathrm{EF}}$ is the axial focusing depth. For numerical evaluation, the element surfaces were discretized at $1 / 3$ of an acoustic wavelength at the highest frequency of interest $(9.0 \mathrm{MHz})$, and the diffraction patterns were computed for $y=0$ [zero-elevation $x z$ plane, Fig. 1(a)] over the same spectral and spatial spans as were used for the measurements described in Section II-C4.

\section{Array Characterization}

1) Ultrasound Array: Time-domain data sets from a linear array (L7-4, ATL Ultrasound, Bothell, WA, USA) with 128 elements of width 0.25 , height 6.5 , and spacing $0.30 \mathrm{~mm}$ were collected using an 256-channel configurable ultrasound array controller (Vantage 256, Verasonics, Kirkland, WA, USA). To support the objectives of this paper, individual array element characterization measurements took two forms: 1) normal incidence sensitivities, used for element response balancing and 2) diffraction patterns describing response variation over a 2-D field of view used to map cavitation.

2) Element Sensitivities: Normal incidence array element sensitivities were determined using a single element unfocused transducer $\left(\mathrm{V} 309,5 \mathrm{MHz}, a_{s}=6.35-\mathrm{mm}\right.$ radius, Olympus NDT, Essex, U.K.) as a source that was scanned over the array aperture from an axial (depth) distance of $z=20 \mathrm{~cm}$. The large standoff provided an incident field that was quasiplanar over the largest (elevation) array element dimension while easing measurement alignment tolerances. The source was driven by a pulser (5072PR, Olympus NDT, Essex, U.K.) triggered by the array controller, and the resulting individual array element responses were digitized and stored. The incident field was separately characterized in the absence of the array using a needle hydrophone $(75-\mu \mathrm{m}$ diameter, Precision Acoustics, Dorchester, U.K.), whose sensitivity was determined in the frequency range of interest through comparison calibration [33] with a membrane hydrophone (DH1602, Precision Acoustics). Hydrophone signals were preamplified (SR 445A, Stanford Research Systems, Palo Alto, CA, USA) before oscilloscope digitization (HDO 4024, LeCroy, Geneva, Switzerland). Hydrophone positioning and oscilloscope data collection were coordinated by control software (UMS2, Precision Acoustics) and synchronized by trigger pulses from the array controller.

Hydrophone scan and array response data sets were processed in MATLAB (Mathworks, Natick, MA, USA) using the following steps: 1) application of a third-order $200-\mathrm{kHz}$ high-pass Butterworth filter for low-frequency noise removal; 
(a)

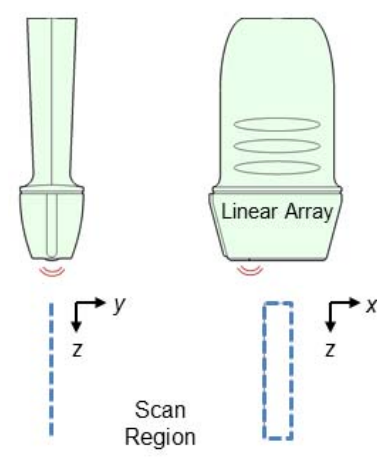

(b)

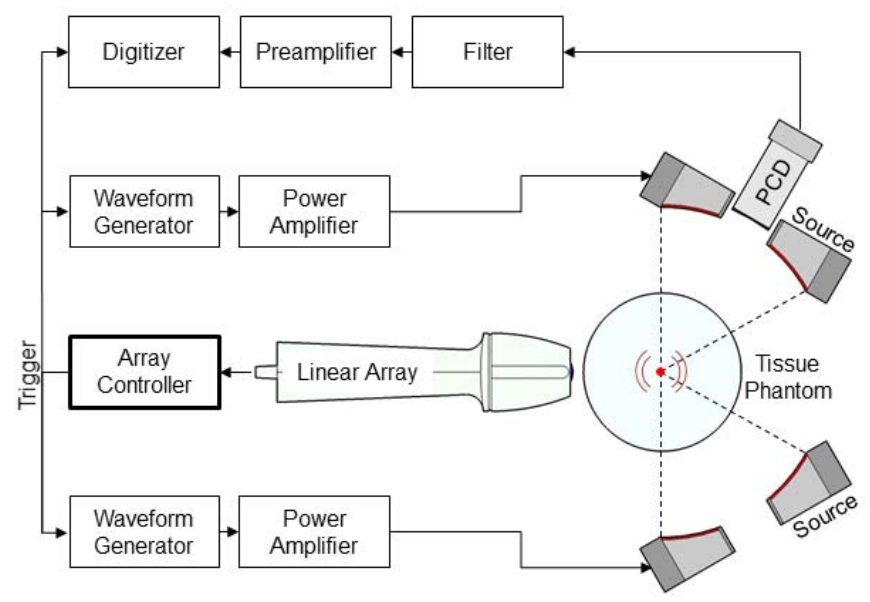

(c)

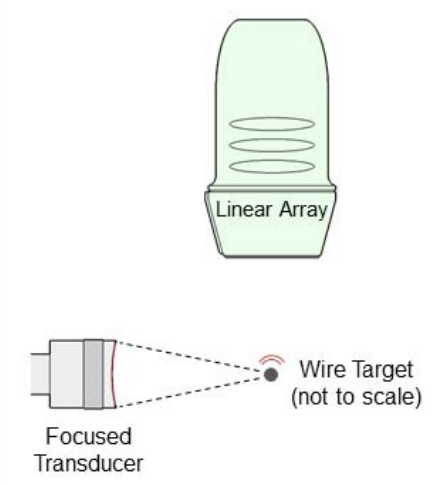

Fig. 1. Experimental configurations for (a) array element diffraction pattern measurements, (b) tissue phantom cavitation monitoring, and (c) validation experiment.

2) application of a $20 \%$ Tukey window to reduce endpoint artifacts in subsequent frequency processing; and 3) calculation of Fourier transforms for each field point and array element. Sensitivities $M_{j}(f)$ for all 128 elements were found from the ratios of the element and hydrophone spectra, the latter being integrated over the element area.

The use of a planar incident field for determination of the element sensitivity allows relatively rapid, high signal-to-noise ratio (SNR) calibration of all array elements. However, the array's focusing lens will decorrelate the phase of an incident plane wave, resulting in a modest low-pass filter effect. This was mitigated through the use of a normal incidence correction factor $C(f)$, found by evaluating (4) at $x=0, z=20 \mathrm{~cm}$, and averaging over $0 \leq y \leq a_{s}$. The correction term was taken to be identical for all elements, and was applied in the final processing of the cavitation data, described in Section II-E.

3) Hydrophone Directivity Measurement: For completeness, the directivity of the needle hydrophone to be used for diffraction pattern measurements (Section II-B4) was measured, rather than using predictions based on an ideal geometry [34]. Measurements were carried out using the same transmission and scanning instrumentation employed for the element sensitivity experiments, with the addition of a manual rotator (PR01, Thorlabs, Ely, U.K.) installed on the positioner. The response of the needle hydrophone was recorded for a range of $-50^{\circ} \leq \theta \leq 50^{\circ}$ (in $5 \pm 0.5^{\circ}$ steps) with respect to normal incidence. These measurements spanned the range of expected arrival angles from all array elements at all distances of interest. The directivity data $Y(f, \theta)$ were stored as spectra normalized by the normal incidence response.

4) Diffraction Pattern Measurement: Individual array element diffraction patterns were determined in transmit mode by driving with a fifteen-cycle linear chirp covering 3-8 MHz, and mapping the resulting pressure field in a rectangular grid spanning -6 to $6 \mathrm{~mm}$ along the array aperture $(x)$ and 25 to $85 \mathrm{~mm}$ in depth (z) [Fig. 1(a)]. Measurement grid steps were 0.5 and $6.0 \mathrm{~mm}$ in the $x$ - and $z$-directions, respectively. The radiated acoustic field was measured with the same needle hydrophone and data collection system as was used for the normal incidence element sensitivity and hydrophone directivity experiments, and signals were averaged 10 times at each scan point. Care was taken to align the scan plane with the center of the array elevation plane $(y=0)$. An additional set of reference field measurements was made to determine the location and on-axis field strength of the elevation focus for each element.

The use of this method proceeded under the assumption that element directivity would be the same whether transmitting or receiving. To enable assessment of this assumption during each individual element transmission, received signals were recorded on the adjoining elements in order to quantify the extent of any electrical crosstalk.

Data sets were processed in the following steps: 1) application of a third-order 200-kHz high-pass Butterworth filter; 2) application of a $20 \%$ Tukey window; 3) calculation of hydrophone $H_{i}(f, x, z)$ Fourier transforms at each field point along the array aperture $(x)$ and depth $(z)$; and 4) calculation of the ratio, here termed "diffraction strength"

$D_{j}(f, x, z)=\frac{H_{j}(f, x, z)}{H_{j}\left(f, x_{\mathrm{ref}}, z_{\mathrm{ref}}\right)} \frac{Y_{j}\left(f, x_{\mathrm{ref}}, z_{\mathrm{ref}}\right)}{Y_{j}(f, x, z)} \frac{R_{j}}{z_{\mathrm{ref}}} e^{i k\left(R_{j}-z_{\mathrm{ref}}\right)}$

where $x_{\text {ref }}$ is laterally centered on each element, $z_{\text {ref }}$ is the axial location of the elevation focus (found from the data to be $32 \mathrm{~mm}), Y_{j}(f, x, z)$ is the needle hydrophone directivity interpolated from $Y(f, \theta)$ using $\theta=\tan (x / z)$. Range estimates were based primarily on propagation time found from a temperature-sound speed relation for water [35], but also included corrections for an observed trigger to transmission delay, and the delay through the external hydrophone preamplifier. The scaling terms at the end of (5) result in $D_{j}$ representing the deviation of the actual diffraction pattern from that of a point source.

Simulations and preliminary experiments indicated that the pressure field patterns changed slowly with respect to element index, particularly near the array center. Full-field measure- 
ments were therefore collected on 14 of the elements $\{1: 3: 16$, $22: 6: 40,52,64,95$, and 128$\}$, and the patterns of the other elements were estimated through spatial interpolation and assumption of left-right symmetry.

\section{Cavitation Measurement}

In order to demonstrate the influence of array diffraction on PAM performance, a set of experiments was conducted wherein cavitation was generated inside a soft tissuemimicking phantom using a pair of source transducers and was monitored with a linear ultrasound array arranged, as shown in Fig. 1(b).

1) Source Transducers: The single-element focused ultrasound (FUS) transducers employed as sources for cavitation generation and monitoring experiments had a fundamental frequency of $1.1 \mathrm{MHz}$, a 64-mm active diameter, and a 62.6-mm spherical focus (H102, Sonic Concepts). The use of a pair of focally overlapping FUS transducers was intended to reduce the axial extent of the pressure field relative to what can be achieved with a single source [36]. The two transducers were driven identically: tone bursts consisting of 25 cycles of $1.1 \mathrm{MHz}$ were output from a waveform generator (model 33250A, Agilent Technologies, Cheshire, U.K.) passed to a power amplifier (ENI A300, Rochester, NY, USA), then on to a matching transformer and the transducer itself. After positioning with a spread angle of $120^{\circ}$ (Fig. 1) and establishing focal overlap, final alignment of the source pair was confirmed through pressure field mapping performed with a needle hydrophone (200- $\mu \mathrm{m}$ diameter, Precision Acoustics). Differences in voltage responses of the two sources were compensated by adjusting waveform generator drive voltage amplitude and delay in order to balance the focal field contributions from each source.

2) Tissue Phantom: A tissue-mimicking phantom was manufactured using a mixture of $0.5 \%$ agar (UltraPure Agarose 1000, Life Technologies, Carlsbad, CA, USA) and $0.25 \%$ talc (Sigma-Aldrich, St-Gallen, Switzerland) by weight in filtered, degassed water [37]. Inclusion of talc was intended to provide cavitation nuclei. Once melted, the agar was poured into a 0.040-mm-thick clear plastic bag lining the interior of a $350-\mathrm{mL}$ glass cylinder and subsequently refrigerated. After setting, the agar bag was removed from the cylinder for acoustic testing. Phantom sound speed, attenuation, and broad spectrum insertion loss of the phantom liner were determined through conventional through-transmission measurements that employed the same source as was used for element sensitivity measurements (Section II-C2), along with a needle hydrophone (200 $\mu \mathrm{m}$, Precision Acoustics).

3) Cavitation Experiments: Cavitation in the tissue phantom was monitored with the L7-4 linear array during FUS exposure, with all system timing coordinated by a synchronization pulse produced once per second from the array controller. Array time series data were digitized without any spectral shaping except for that which was provided by the controller's antialiasing filter, and stored for subsequent analysis. Each array data record was $180 \mu \mathrm{s}$ in duration, sufficient for capturing any acoustic emissions created in the phantom by the
22.7- $\mu$ s cycle FUS pulse. The data record contained an initial window of no less than $60 \mu \mathrm{s}$ of background noise prior to the arrival of cavitation signals and FUS source sidelobe radiation. This signal-free window was used to calculate sensor SNR for each element.

Measurements were made with array offset distances of $z=30-80 \mathrm{~mm}$ with respect to the FUS source focus, with 10-20 FUS pulse exposures ("shots") recorded per offset. Moving the array in this manner allowed investigation of processing and beamformer performance as a function of distance in the 0-elevation plane [xz, Fig. 1(a)], and mimics possible therapeutic monitoring scenarios where cavitation activity may be produced by a source moving through a broad range of locations. Since the phantom cavitation response weakened after several excitation pulses at a single location, measurements were repeated after shifting the phantom position in order to interrogate a "fresh" region.

An independent observation of cavitation activity was made using a single element focused transducer (Panametrics V320, $7.5 \mathrm{MHz}, 2.95$ " spherical focus, Olympus NDT) acting as a passive cavitation detector (PCD) [38]. The PCD was coaxially mounted in the center hole of one of the two H102 sources so that cavitation activity could be monitored at a fixed location while the array and phantom positions were varied. PCD element data were high-pass filtered (F5081-2P0, Allen Avionics, Mineola, NY, USA), preamplified (SR445A, one stage), and digitized (HS3, TiePie Engineering, Netherlands) upon triggering from the array controller. The purpose of the PCD data was limited to temporal monitoring of relative cavitation emission strength, so no calibration was performed.

\section{E. Diffraction and Sensitivity Correction}

Individual element corrections were applied to raw array cavitation monitoring data in the frequency domain for each location in the imaging plane to which the beamformers were steered. Processing steps consisted of: 1) application of a $20 \%$ Tukey window to the time-domain array data $S_{j}(t)$ in order to capture cavitation events and minimize the cumulative level of electronic noise; 2) Fourier transformation $\left[X_{j}(f)\right]$ of the windowed array data; 3 ) application of sensitivity and diffraction correction terms; and 4) inversion of the spectra back to the time domain for use in existing beamformer codes

$$
\begin{aligned}
p_{j, \text { comp }}(t) & =L\left\{\operatorname{Re}\left[\operatorname{IFT}\left(X_{j}(f) /\left(D_{j}(f, x, y) M_{j}(f) C(f)\right)\right]\right\}\right. \\
p_{j, \text { uncomp }}(t) & =L\left\{\operatorname{Re}\left[\operatorname{IFT}\left(X_{j}(f) /\left(M_{j}(f) C(f)\right)\right)\right]\right\} .
\end{aligned}
$$

The subscripts comp and uncomp indicate whether diffraction corrections were applied, IFT indicates an inverse Fourier transform, $\operatorname{Re}[$ ] indicates the real part of the argument in braces, and $L\{\}$ is a low-pass filtering operation described later. Equation (6) shows that each channel of the array response data is modified by a spatially independent element sensitivity $\left[M_{j}(f) C(f)\right]$, and a diffraction strength term $D_{j}(f, x, y)$ that depends on frequency, range, and angle between each element and each point in the imaging plane. 
At short distances and high frequencies, the diffraction patterns may have minima or nulls that would amplify the sensor noise when applied in (6). To mitigate this problem, the diffraction terms $D_{j}$ were prewhitened with the addition of a random noise floor term with rms amplitude of 0.01 relative to the array maximum value of $D_{j}(\sim 1)$. Low-pass filter corner frequencies were set by consideration of both datainvariant and data-specific metrics for each cavitation experiment data set. First, the lowest frequency $f_{j, 1}(x, y)$ at which the whitened $D_{j}$ term fell below 0.2 was determined for each element. Next, the lowest frequencies for which the individual element SNRs fell below $3 \mathrm{~dB}$ were found $\left(f_{j, 2}\right)$. Low-pass filter corner frequencies for use in (6) and (7) were chosen to be the minimum of $\left[f_{j, 1}(x, y), f_{j, 2}\right]$, and filter coefficients were then calculated using a fifth-order Butterworth formulation. The time series in (6) and (7) were run through the filter both forward and backward in order to remove passband phase distortion. Applying the same filtering operations to both data sets ensured consistency of bandwidth for subsequent performance comparisons. The corrected and filtered time series $p_{j, \text { comp }}(t)$ and $p_{j \text {,uncomp }}(t)$ were then passed to the TEA and RCB beamformers.

\section{F. Validation Experiment}

A validation experiment was conducted in order to assess the accuracy of the calibration procedures described in the preceding sections. Ideally, the validation source would have dimensions of an order consistent with typical cavitation events to be monitored with PAM, and be time stable so that the emitted field could be carefully characterized. To meet these criteria, a wire scattering configuration (see [5], [6]) was employed [Fig. 1(c)]. A 10-cm-long Tungsten wire (Goodfellow Cambridge Limited, Huntington, U.K.) of 0.10-mm diameter was held taut in a clear plastic fixture (not shown) and irradiated at the focus of a broadband (3-7 MHz) transducer with a 0.9 -mm full-width half-power beam at $5 \mathrm{MHz}$ (V380SU, 1.0" diameter, Olympus NDT, Essex, U.K.). The use of a large diameter source transducer and small diameter wire were intended to cumulatively minimize the directivity of the scattered field.

The wire-scattered field was monitored in turn by the L7-4 array and the calibrated $75-\mu \mathrm{m}$ diameter needle hydrophone, the latter being scanned over a line spanning the aperture of the array. All measurements were conducted with a $55-\mathrm{mm}$ nominal offset from the wire, corresponding to the middle of the range of typical cavitation imaging work with the L7-4. The pulser used for element sensitivity measurements (Section II-C2) was used both to drive the focused transducer and to filter and preamplify the relatively small signals from the needle hydrophone. The preamplification gain and phase were determined as a function of frequency using a reference signal provided by a waveform generator (single $5-\mathrm{MHz}$ cycle from 33250A, Agilent Technologies) and calculating the spectral ratio of amplified and unamplified signals. Wire backscatter measurements were periodically acquired on the focused transducer as a measure of alignment and drive level stability.
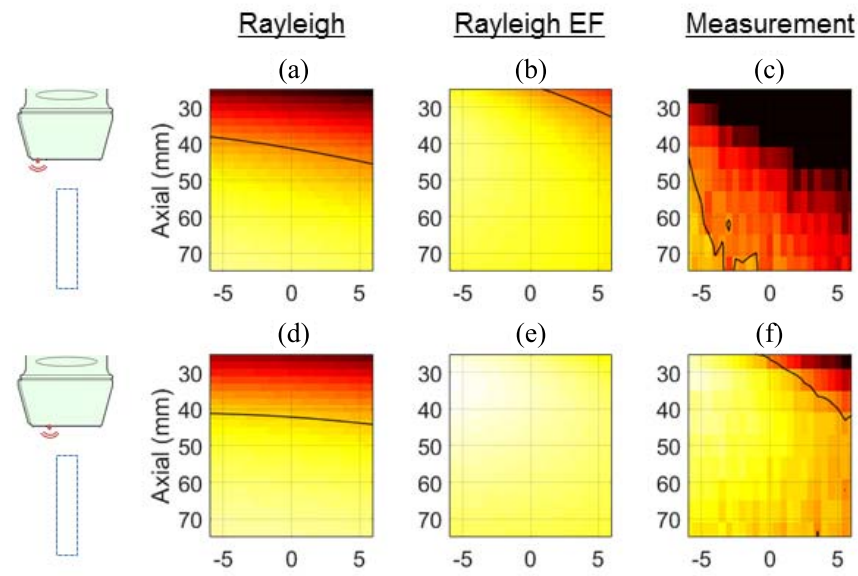

(e)
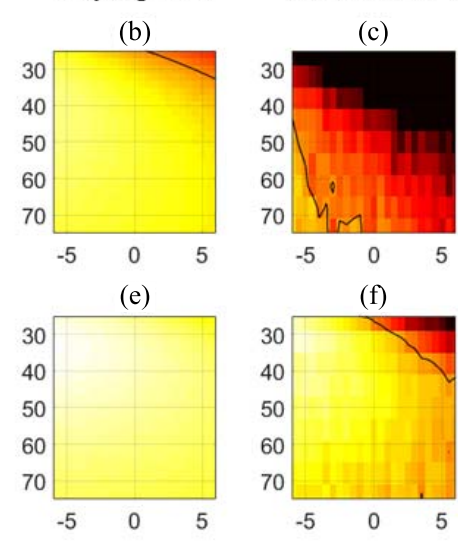

(f)

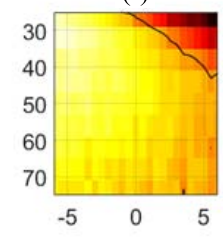

(g)
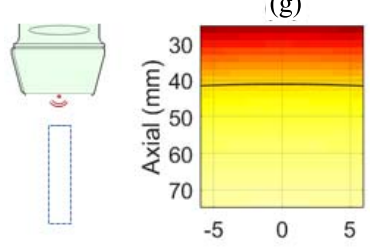

(h)

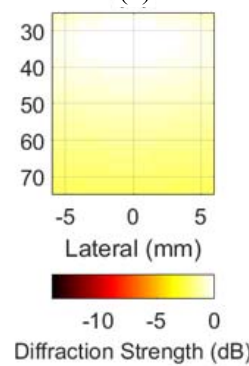

(i)

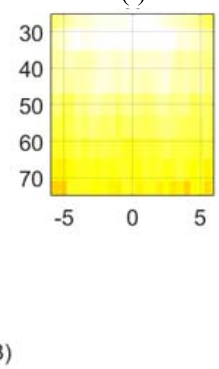

Fig. 2. Comparison of the Rayleigh integral calculations ("Rayleigh," left column), Rayleigh integral calculations with 32-mm elevation focus ("Rayleigh EF," middle column), and measurements (right column) of the diffraction pattern for (a)-(c) array element 1, (d)-(f) element 34, and (g)-(i) element 64 at a frequency of $6 \mathrm{MHz}$. Element positions are illustrated to the left of the data plots. The black line overlay indicates a $-6-\mathrm{dB}$ contour.

Array signals were windowed to exclude direct path signals from the focused transducer and processed according to (6) to yield element pressures. Hydrophone signals were corrected for sensitivity, directivity, and preamp gain $G_{\text {pre }}(f)$ according to

$$
\begin{aligned}
p_{\text {hyd }}(t, x, z) & =L\{\operatorname{Re}[\operatorname{IFT}(X(f, x, z) / K(f, x, z))]\} \\
K(f, x, z) & =M_{\text {hyd }}(f) Y(f, x, z) G_{\text {pre }}(f) .
\end{aligned}
$$

In all cases, the voltage spectra $X$ were calculated as in Section II-E, and the filter $L$ was set with a corner frequency of $7.0 \mathrm{MHz}$.

\section{RESUlTS}

\section{A. Array Element Diffraction Patterns}

Examples of measured array element diffraction patterns are shown in Fig. 2 for a frequency of $6.0 \mathrm{MHz}$ (near the center of the 3.5-8.0 MHz band where sensitivity and diffraction data were both available), along with pattern predictions described in Section II-B. The displayed quantity in Fig. 2, referred to as "diffraction strength," is the diffraction pattern scaled by the distance from the array element to each field point (5). A value of $0 \mathrm{~dB}$ indicates that the element responded as if it were a point monopole. The measurements show much stronger position dependence than either of the predictions, but the inclusion of an elevation focus leads to improved 

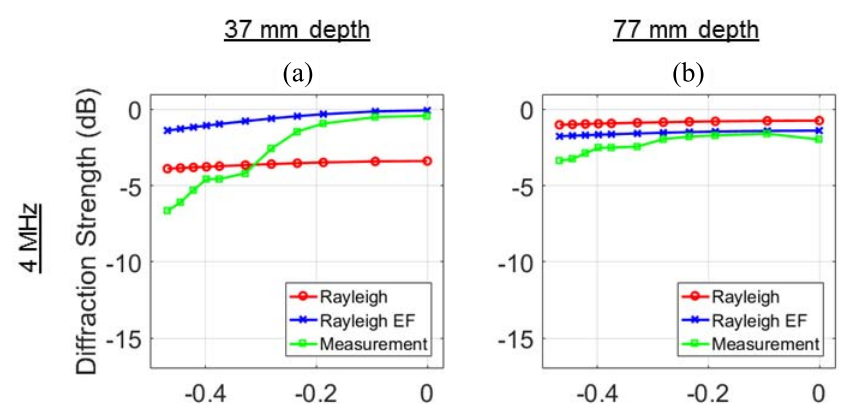

(c)
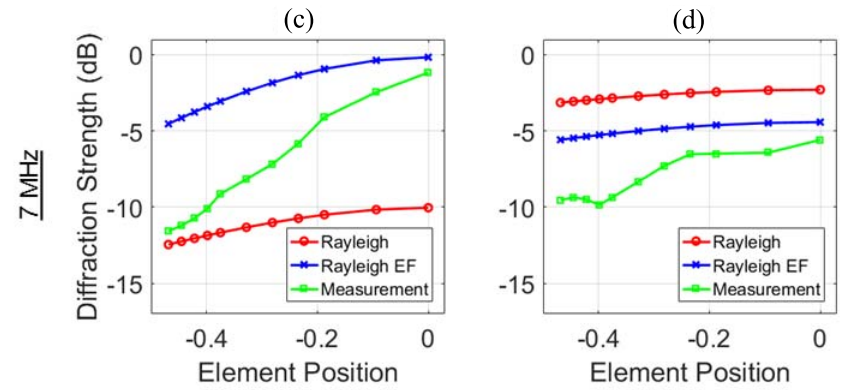

Fig. 3. Predicted and measured diffraction patterns for elements spanning the left half of the array, observed along the array centerline (between elements 64 and 65). Four-megahertz patterns for axial distances of (a) 37 and (b) $77 \mathrm{~mm}$. Seven-megahertz patterns for axial distances of (c) 37 and (d) $77 \mathrm{~mm}$. Element positions are normalized relative to the full array aperture, so the left half of the array spans a range of -0.5 to 0.0 .

agreement, particularly when the angle between element and field point is small, as shown in Fig. 2(h) and (i).

The variation of diffraction strength with frequency, axial distance, and element position is shown in Fig. 3 along the centerline of the array (between elements 64 and 65) for measured elements spanning the left half of the array aperture. As expected, diffraction effects are strongest at the high frequency and shortest axial range [Fig. 3(c), $7 \mathrm{MHz}$, $37 \mathrm{~mm}$ ]. Best agreement with measurements is achieved when the prediction includes an elevation focus, the angle between array element and field point is smallest (near-center elements, long axial range), and at lower frequencies [see Fig. 3(b)]. These are the same conditions under which deviations from point monopole behavior are minimal (diffraction strength $\sim 0 \mathrm{~dB}$ ). Collectively, Figs. 2 and 3 demonstrate that neither a point monopole nor the 2-D models employed here is adequate for describing the array element responses under many PAM application conditions.

\section{B. Calibration Validation}

Before describing the impact of using measured diffraction data to compensate array-measured cavitation signals, it is important to confirm that the proposed calibration approach is valid for a "known" source. The results of the validation experiment are summarized in Fig. 4. Root-mean-square pressure $\left(p_{\mathrm{rms}, j}\right)$ scattered by the thin-wire target is shown as a function of position (either for the fixed array elements or the scanning hydrophone), all calculated within a $\tau=10-\mu$ s time window. The element diffraction compensation process modifies the array pressure distribution so as to closely match the

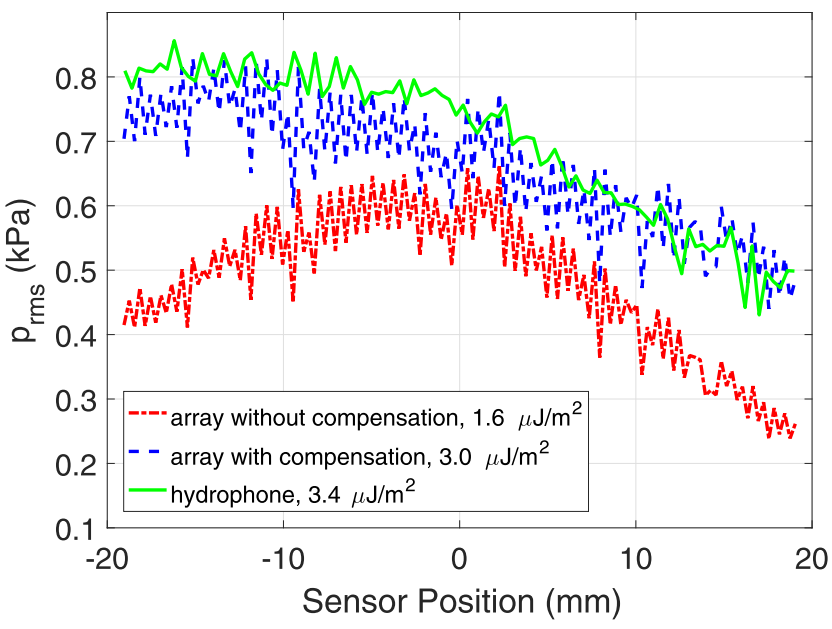

Fig. 4. Array and scanned hydrophone rms pressures obtained from the validation experiment. Negative position values denote locations in the same half space as the focused transducer illuminating the target. Values in the legend are spatially averaged energy per unit area.

absolute amplitudes observed with the scanned hydrophone. Without compensation, the array field is substantially distorted. Asymmetry of the calibrated fields is generally consistent with expectations for side scattering from a cylinder whose radius is acoustically small [39].

The array response data in Fig. 4 indicate a larger degree of point-to-point variability than the hydrophone scan. The scale of this variability appears to be on par with the cumulative receive and transmit channel sensitivity standard deviations ( $8 \%$ and $3 \%$, respectively) after calibration, which themselves are similar to the rated values for array controller variability (12\% and $3.5 \%$, respectively) [40].

Values of spatial average energy per unit area $(F=$ $\left.(\tau / N \rho c) \sum_{j=1}^{N} p_{\text {rms }, j}^{2}\right)$ shown in the legend of Fig. 4 indicate that the diffraction compensation process raised the array estimate from $47 \%$ to $88 \%$ of the hydrophone estimate. The final discrepancy of $12 \%$ is the same size as the bestcase uncertainty for the hydrophone calibration (1.06, squared for energy estimates). These results validate the calibration and correction procedures described in Sections II-C and II$\mathrm{E}$, and facilitate quantitative analysis of cavitation maps in Sections III-C-III-F.

\section{Effect of Diffraction Correction on Cavitation Energy Estimation}

The impact of array element response compensation on cavitation mapping is shown in Fig. 5 for TEA and RCB beamformers applied to tissue phantom cavitation events centered at an axial observation offset (depth) of approximately $57 \mathrm{~mm}$. Despite the stark differences in map patterns between the two beamformers, the net effect of the compensation processing is seen to be similar for both in terms of cavitation energy estimation. The corrected map energies are elevated on the order of 3-5 dB (factor of 2-3), with a relatively modest impact on beamwidth ( $<15 \%$ both axially and laterally). These results demonstrate the importance of diffraction correction for obtaining comparable cavitation measurements irrespective of the beamforming algorithm used. 


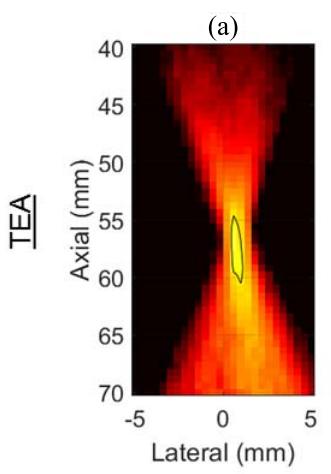

(d)

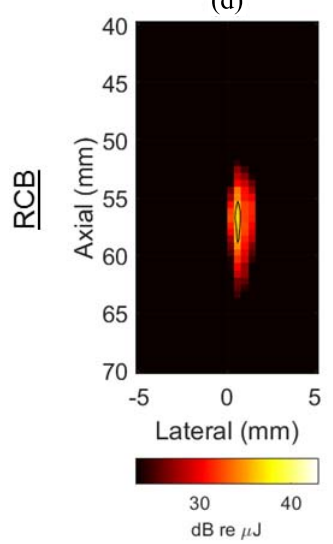

(b)

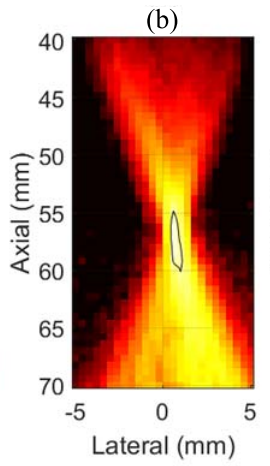

(e)

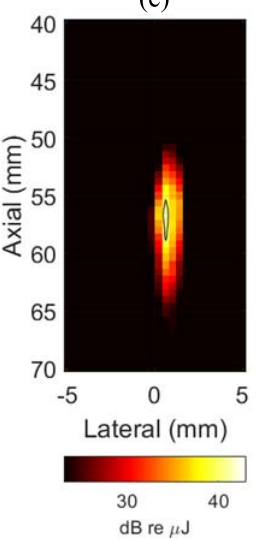

(c)

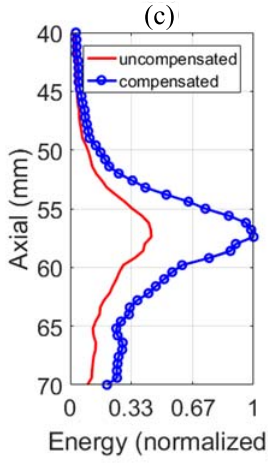

(f)

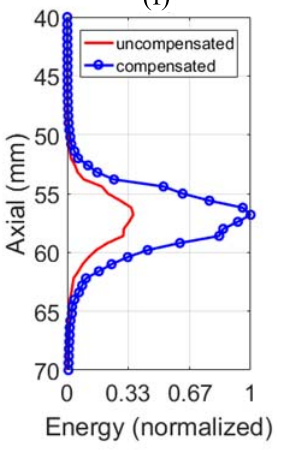

Fig. 5. Examples of passive acoustic maps of a single ultrasound shot observed with an array offset of approximately $57 \mathrm{~mm}$. TEA beamformer (a) uncompensated, (b) compensated, and (c) axial patterns passing through the image maxima. RCB beamformer (d) uncompensated, (e) compensated, and (f) axial patterns passing through the image maxima. The thin-black line contour overlays on the cavitation images indicate $70 \%$ amplitude boundaries for each map. (a)-(e) have identical color scales. Values in (a) and (c) were normalized by the compensated image maximum in (b), while (d) and (f) were normalized by the image maximum in (e).

\section{Beamformer Sensitivity to Diffraction Correction}

The influence of diffraction compensation on beamformer performance as a function of depth is shown in Fig. 6. The ratios of uncompensated and compensated peak energies indicate how strongly the map maxima are changed by the diffraction compensation process. Under ideal conditions, the ratios would further indicate the extent of energy under estimation (i.e., estimation error) if diffraction were not accounted for. The green line in Fig. 6 is the mean square of the measured diffraction strength (MSD) at three depths along the array centerline $(x=0)$, integrated over the $\Delta f=3.5-7.0 \mathrm{MHz}$ band

$$
\operatorname{MSD}(z)=\frac{1}{N} \frac{1}{\Delta f} \sum_{j=1}^{N}\left(\int_{f=3.5}^{f=7.0}\left|D_{j}(f, x=0, z)\right| d f\right)^{2} .
$$

The TEA beamformer mean energy ratios vary between 0.38 and 0.5 , meaning that in the absence of any compensation, the algorithm would typically underestimate the energy by more than a factor two. The TEA values track the MSD curve within 10\%-20\%, suggesting that MSD may be used as a quick estimate of diffraction effects on the peak energy calculation when the cavitation activity is near the array centerline.

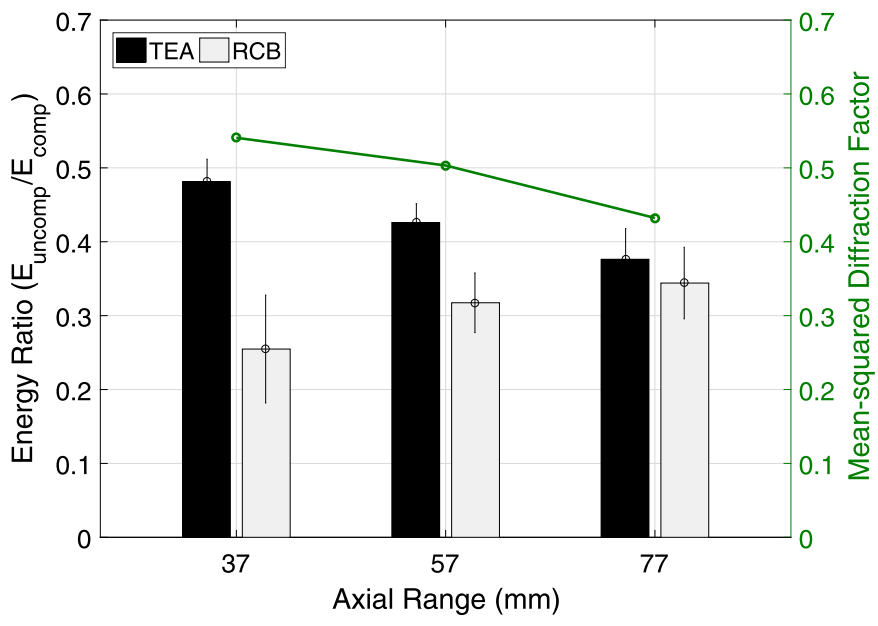

Fig. 6. Ratios of peak cavitation map energy without ( $\left.E_{\text {uncomp }}\right)$ and with ( $\left.E_{\text {comp }}\right)$ diffraction compensation as a function of beamformer type and axial range between cavitation events and the receive array. Solid bar heights indicate multiple shot averages $(N=15-20)$ and error bars indicate \pm 1 standard deviation. The green overlay (right ordinate) is the mean-squared diffraction strength integrated from 3.5 to $7.0 \mathrm{MHz}$.

By contrast, the RCB results show a more pronounced sensitivity to imaging depth, with strongest effects at short distances. Insight into this behavior may be gained from examination of the RCB-calculated array weights as shown in Fig. 7. The examples shown are for two cavitation events observed at axial ranges of 37 and $77 \mathrm{~mm}$, with weights displayed for the image pixel corresponding to the location of image peak energy. The RCB process tends to reduce the central element contributions and elevate those of the end elements in order to improve resolution and suppress influence from cavitation events in nearby locations.

At the shorter axial range $(37 \mathrm{~mm})$, the uncompensated weights show a broader suppressed central region and narrower end element amplification, meaning that the beamformer is relying on a relatively small number of elements to adjust the overall response. These end elements also show the strongest diffraction effects, namely, loss of bandwidth and mean-square signal level. Upon compensating the array elements, the variance of the weights is seen to significantly decrease.

Although the two cavitation events in this example were not identical (recorded nonsimultaneously at two depth offsets), they serve to illustrate how RCB operates on array channel data, and why short range energy ratios (Fig. 6) would show more pronounced compensation effects.

\section{E. Effect of Diffraction Correction on Cavitation Map Spatial Resolution}

The impact of diffraction compensation on cavitation map half-amplitude full-axial beamwidth is shown in Fig. 8. The TEA results exhibit a beamwidth decrease on the order of 10\%-15\% after compensation (black and light gray bars), while the RCB results indicate a negligible decrease (dark gray and white bars). Similar results were seen for lateral beamwidth (data not shown). Together these results raise questions about how different beamformers make use of broadband 
(a)

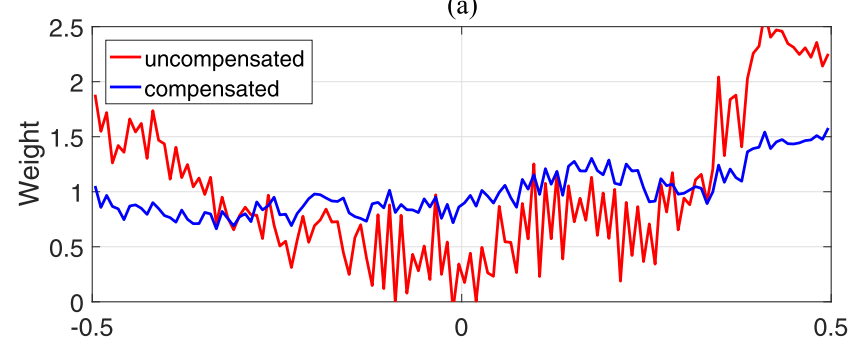

(b)

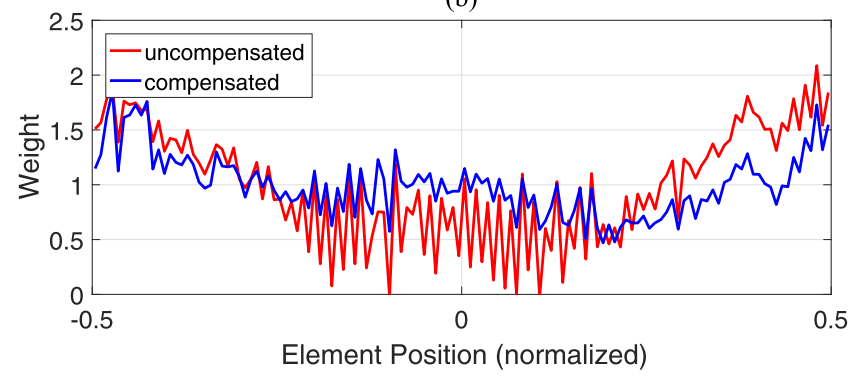

Fig. 7. RCB weights for cavitation events centered at depths of (a) 37 and (b) $77 \mathrm{~mm}$. Weight values are shown for image pixels corresponding to the location of maximum estimated energy. Element positions are normalized relative to the full array aperture.

information to form an image, but this issue is beyond the scope of this paper.

Overall, the diffraction compensation effects on beamwidth are relatively small relative to the differences in absolute beamwidth attained with the two beamformer methods. This implies that resolution of broadband cavitation emissions was limited by the low end of the received signal spectrum, which was not substantially altered by the diffraction compensation process. As such, PAM resolution of inertial cavitation events may be improved simply by applying a high-pass filter to the array data [5], [6]. Moreover, the weights found with the current implementation of RCB are frequency-independent and real-valued, and it may be that the absence of these restrictions would allow better utilization of restored high frequency information to further improve resolution. Assessment of the tradeoff between bandwidth, resolution, and interfering source suppression is beyond the scope of this paper, and will be treated as part of a subsequent paper.

\section{F. Effect of Diffraction Correction on Beamformer Bias}

Up to this point, the impact of diffraction correction has been described with a mixture of absolute and relative energy metrics, and a question remains whether the proposed processing approach is likely to mitigate potential observation biases, particularly as related to the use of different beamformers. Fig. 9 shows map-peak cavitation energy estimates for eight consecutive shots recorded at a depth of approximately $32 \mathrm{~mm}$. For each shot, the individual bars show peak map energies for both beamformers, before and after diffraction compensation. The green overlay quantifies energy calculated with the PCD, which served as an independent estimator of cavitation activity.

The energy levels observed by the PCD and the beamformed array drop more than an order of magnitude over the shot sequence, perhaps as expected for a phantom (with

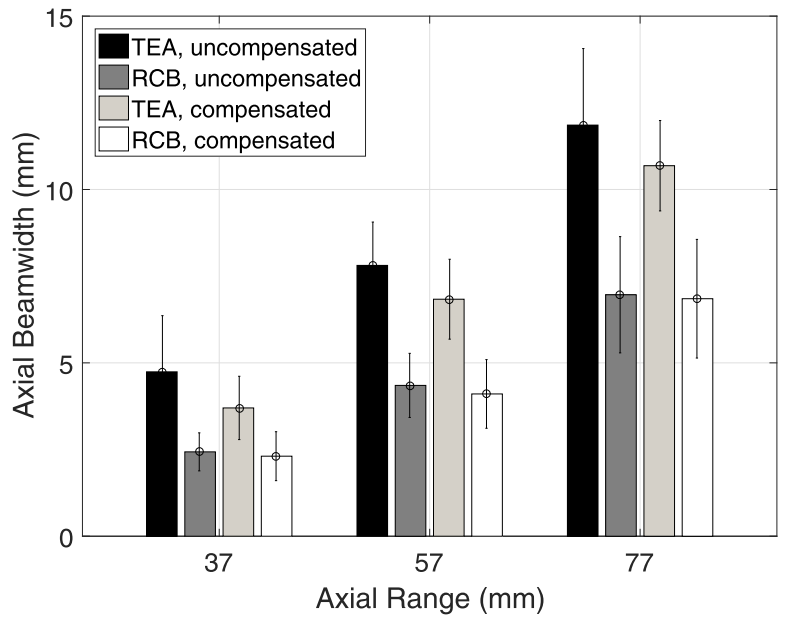

Fig. 8. Half-amplitude full-width cavitation map axial beamwidths without and with diffraction compensation as a function of beamformer type and cavitation depth. Solid bar heights indicate multiple shot averages $(N=15-20)$ and error bars indicate \pm 1 standard deviation.

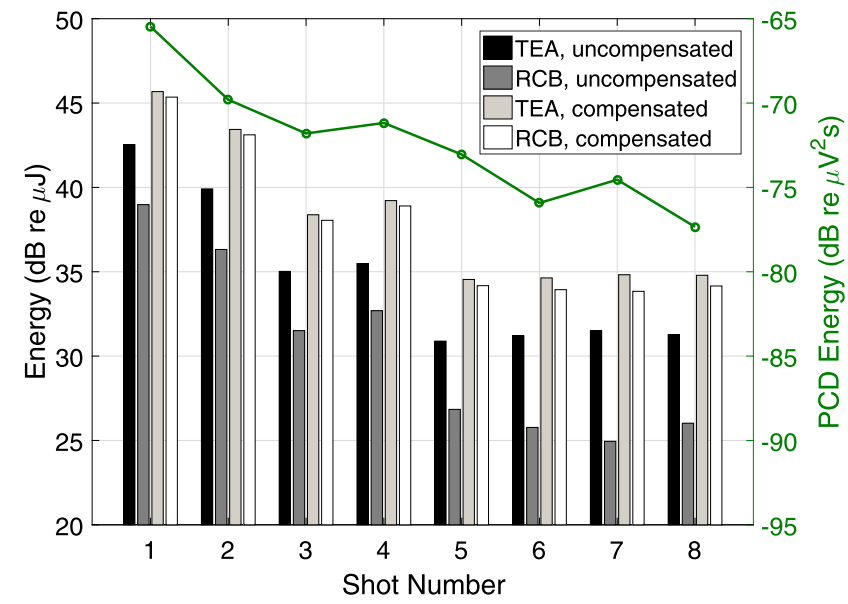

Fig. 9. Energy estimates from the array (solid bars) and PCD (green line) for eight shots recorded when the array was approximately $32 \mathrm{~mm}$ from the cavitation activity. Both energy axes have $30-\mathrm{dB}$ range. The PCD transducer was not calibrated, and is therefore displayed in voltage units.

no flow or other means to replenish nuclei) being repeatedly exposed at the same location to FUS at an incident pressure level close to the cavitation threshold (approximately 1.5-MPa peak negative). As the energy levels dropped in both measurement systems with increasing shot number, the PCD and array results diverged somewhat. This is not surprising, since the PCD and the linear array have different spatial sensitivity patterns, and the cavitation activity is not necessarily confined to the PCD focus.

The uncompensated peak energies estimated with the two beamformers show discrepancies (black and dark gray bars) that increase from a factor of approximately two to as much as five as the overall energies dropped. However, the diffraction compensation process largely erased these discrepancies, with the two compensated beamformers (light gray and white bars) yielding values within $12.2 \% \pm 6.6 \%$ (mean \pm standard deviation) of each other over the ensemble of shots. Echoing the results in Fig. 6, the compensation process always has a larger effect on RCB-computed energies than on those of TEA. The compensated RCB energies are slightly smaller than 
those of TEA-a result which is thought to be a consequence of $\mathrm{RCB}$ rejection of interfering regions that may artificially inflate TEA estimates [7].

\section{Discussion}

The preceding results demonstrate the significance of diffraction correction for PAM, particularly when imaging cavitation events relatively close to the monitoring array. For such measurement scenarios (see [7], [41]), this paper implies that passive mapping studies performed to date may have substantially misreported cavitation source energies. One interpretation may then be that it would be simplest to not use those array elements that are farthest from the imaging location of interest. A predefined subarray beamforming approach [5] is one such example, but the tradeoffs between reduced spatial resolution and reduced diffraction sensitivity would need to be carefully weighed against the needs and priorities of the beamforming activity (e.g., detection, localization, and energy quantification). As presented here, the diffraction correction process appears to provide more flexibility for how the array aperture may be used.

SNR-based selection of array channels, in combination with an adaptive beamformer, may ultimately prove to provide the best resolution while mitigating the influence of noisy data near the aperture boundaries. Regardless of the aperture chosen, some degree of amplitude correction relative to beamformer-presumed diffraction would appear to be necessary for mapping over a broad range of axial positions and frequencies.

All diffraction terms were measured in transmission mode and used under the assumption that the spatial field characteristics are the same whether the elements are used as transmitters or receivers. The drive level used for these experiments was approximately $40 \%$ of the full range of the drive system, and spot checks at lower drive levels did not reveal any changes in element response detectable beyond the measurement uncertainty associated with finite SNR. This suggests that nonlinearity in the electromechanical response of array structure was unlikely to influence the results, but it does not rule out electrical or mechanical interelement coupling. However, the responses of elements immediately adjoining a driven element showed no signal until approximately $0.33 \mu \mathrm{s}$ after the transmission began (data not shown). This delay is on a plausible scale for intra-array wave propagation [e.g., the time to travel across one element $(0.3 \mathrm{~mm})$ through a slow lens-like material $(c \sim 910 \mathrm{~m} / \mathrm{s})]$. Based on these observations, electrical crosstalk did not appear to impact the diffraction measurements.

No simple scaling of the element width was found to provide a suitable diffraction pattern match over the range of frequencies and observation points in this paper. A more complete model incorporating lens refraction, losses, and other aspects of array construction may well reproduce the salient field features, but development of such models still requires validation through experiment.

Uncertainties enter the array compensation process from multiple sources that may be relatively straightforward (hydrophone and array controller calibration) or challenging (component alignment) to quantify. As such, one added value of the validation experiment is to quantify the cumulative error. In this paper, the array energy estimate was $12 \%$ below that which was based on the hydrophone scan. Whether this level of uncertainty should be expected from the proposed procedures will not be known until they are repeated several times with multiple arrays and acquisition systems.

This paper featured a single serial number of one array model, so it is natural to question whether other arrays would show similar behavior. Field pattern measurements made with two other linear arrays (Verasonics L11-4v, ATL L12-5, data not shown) showed trends relative to the L7-4 that were consistent with general expectations based on element size and elevation focus.

Alternative methods for assessing element diffraction effects include small target scattering measurements [6], [42], [43], performed over suitable spatial and spectral ranges. While perhaps more challenging based on the small signal levels in such experiments, there may be advantages in that all element responses could be obtained from the same data set, and the procedure of field normalization at the elevation focus would be unnecessary. Based on the SNR observed in the validation experiment (Section II-F), array characterization over the relevant range of target locations would take several weeks of facility time. By contrast, the calibrations described in Section II-C were completed in two days.

Since diffraction patterns were measured in a plane normal to and centered on the array aperture, no elevation plane integration effects were included in the correction calculations. This was intentional, as the significance of elevation directivity would depend on the spatial distribution of cavitation in the elevation plane, which is generally not known and is FUS-system dependent. Spot checks indicated that elevation directivity effects could be nonnegligible at high frequencies and short ranges near the elevation focus, but quantifying the impact will require further study.

The energy values determined by the TEA and RCB beamformers are based on partial spatial sampling of the cavitation field with a diagnostic array. Formally, the radiated acoustic energy should be found from the intensity integrated over a surface that completely encloses the source(s) [32]. The beamformers have been numerically validated for simple cases [44], and quantification of energy estimation uncertainties arising from the use of partial field sampling is part of ongoing research.

The various filtering and inversion processes described here were carried out for every location in the imaging domain. Processing may be greatly accelerated by running the beamformer once without corrections in order to locate the primary cavitation region, then a second time in order to apply corrections over a limited region of interest. Independently, faster algorithmic implementation will be possible through the use of frequency-domain beamforming, where retained data volumes are smaller [8], filtering operations need not be causal, and multiple inversions (time to frequency to time) of element data need not be done at all. All of these approaches may be further accelerated through the use of graphical processing units [8], [45]. 
Array element SNR during the cavitation measurements rarely exceeded $30 \mathrm{~dB}$, largely as a consequence of operating the excitation sources to produce an incident pressure just above the inertial cavitation threshold of the agar phantom. The modest SNR, particularly on the array edge elements in the short range experiments described in Section III-F, was useful in illustrating methods to keep inversion processing from significantly enhancing noise contamination of the energy estimates. Noise contamination of PAM data manifests as elevated sidelobe and "tail" levels [7], neither of which were seen in the analyses. However, since all tests presented here were conducted in low-loss media (phantom attenuation $<0.2 \mathrm{~dB} / \mathrm{cm}$ at $9 \mathrm{MHz}$, data not shown), limitations on application of the diffraction correction technique in terms of useable bandwidth are not yet known. For clinically relevant soft tissue applications, short range (less than the elevation focus) measurements may still be dominated by diffraction effects, while at longer ranges, diffraction effects may be dwarfed by path attenuation. Accounting for both, along with related analysis of range-related mapping biases are the subjects of our next paper.

The array diffraction terms were measured in a water tank at a temperature of approximately $19{ }^{\circ} \mathrm{C}-20{ }^{\circ} \mathrm{C}$, where the sound speed in water and the phantom were estimated to be within $5 \mathrm{~m} / \mathrm{s}$ of each other. Sound speeds in clinically relevant soft tissues may differ from the water on the order of $\pm 10 \%$ [46]. The influence of tissue sound speed and its spatial distribution on diffraction correction is expected to be modest given the slow variations in the image plane observed in this paper, but the cumulative effects on energy estimation and image resolution are the subjects of ongoing research.

\section{CONCLUSiON}

The results in this paper highlight the significance of experimentally characterizing array element responses for accurate quantitative mapping of cavitation sources. Diffraction correction is particularly important when: 1) comparing cavitation data from different depths, arrays, or processed with different beamformers or 2) using absolute cavitation thresholds to indicate a particular bioeffect. Both point and planar 2-D element diffraction predictions were found to provide inaccurate estimates of the measured fields over a large range of relevant conditions, with limited improvement gained by including an elevation focus. Discrepancies grew as frequency and image plane angle increased, and it appears that measurement-based element characterization is necessary for accurate array modeling and processing, as well as for validation of any numerical methods applied to the imaging problem being addressed.

The diffraction compensation process, as validated here with a small target scattering experiment, serves to rebalance the spectrum of each array element, and in combination with a sensitivity calibration, produces element responses which should be unbiased by the specifics of the element geometry and construction. This in turn is intended to remove array and depth related biases from cavitation mapping processing - an essential part of comparing results from different beamformers, laboratories, or clinics. Quantitatively, compensation for measured diffraction effects manifested as an increase in estimated cavitation energy by as much as a factor of five, while eliminating a beamformer algorithm bias. Absolute or at least consistent estimation of cavitation energy may be of critical concern in applications where a certain amount of bubble activity is correlated with a desired bioeffect. In other applications where detection of cavitation is paramount, diffraction compensation processing may be less important. Although examined in the context of PAM for cavitation monitoring, the techniques used here may also be applied to B-mode or active cavitation monitoring, potentially offering more accurate and improved imaging results than when assuming ideal point or plane radiator directivity.

\section{ACKNOWLEDGMENT}

The authors would like to thank D. Elbes and C. Mannaris for their helpful discussions and assistance with instrumentation.

\section{REFERENCES}

[1] J. J. Kwan et al., "Ultrasound-propelled nanocups for drug delivery," Small, vol. 11, no. 39, pp. 5305-5314, Aug. 2015.

[2] M. A. O'Reilly, R. M. Jones, and K. Hynynen, "Three-dimensional transcranial ultrasound imaging of microbubble clouds using a sparse hemispherical array," IEEE Trans. Biomed. Eng., vol. 61, no. 4 pp. 1285-1294, Apr. 2014.

[3] J. T. Sutton, K. J. Haworth, G. Pyne-Geithman, and C. K. Holland, "Ultrasound-mediated drug delivery for cardiovascular disease," Expert Opin. Drug Del., vol. 10, no. 5, pp. 573-592, May 2013.

[4] M. Gyongy, M. Arora, J. A. Noble, and C. C. Coussios, "Use of passive arrays for characterization and mapping of cavitation activity during HIFU exposure," in Proc. IEEE Ultrason. Symp., Beijing, China, Nov. 2008, pp. 871-874.

[5] V. A. Salgaonkar, S. Datta, C. K. Holland, and T. D. Mast, "Passive cavitation imaging with ultrasound arrays," J. Acoust. Soc. Amer, vol. 126, no. 6, pp. 3071-3083, Dec. 2009.

[6] M. Gyöngy and C.-C. Coussios, "Passive spatial mapping of inertial cavitation during HIFU exposure," IEEE Trans. Biomed. Eng., vol. 57, no. 1, pp. 48-56, Jan. 2010.

[7] C. Coviello et al., "Passive acoustic mapping utilizing optimal beamforming in ultrasound therapy monitoring," J. Acoust. Soc. Amer., vol. 137 , no. 5, pp. 2573-2585, May 2015.

[8] K. J. Haworth, K. B. Bader, K. T. Rich, C. K. Holland, and T. D. Mast, "Quantitative frequency-domain passive cavitation imaging," IEEE Trans. Ultrason., Ferroelect., Freq. Control, vol. 64, no. 1, pp. 177-191, Jan. 2017

[9] C. D. Arvanitis, C. Crake, N. McDannold, and G. T. Clement, "Passive acoustic mapping with the angular spectrum method," IEEE Trans. Med. Imag., vol. 36, no. 4, pp. 983-993, Apr. 2017.

[10] J. Gateau, J.-F. Aubry, M. Pernot, M. Fink, and M. Tanter, "Combined passive detection and ultrafast active imaging of cavitation events induced by short pulses of high-intensity ultrasound," IEEE Trans. Ultrason., Ferroelect., Freq. Control, vol. 58, no. 3, pp. 517-532, Mar. 2011.

[11] T. Li, T. D. Khokhlova, O. A. Sapozhnikov, M. O’Donnell, and J. H. Hwang, "A new active cavitation mapping technique for pulsed HIFU applications-bubble Doppler," IEEE Trans. Ultrason., Ferroelect., Freq. Control, vol. 61, no. 10, pp. 1698-1708, Oct. 2014.

[12] T. Ding et al., "Spatial-temporal three-dimensional ultrasound plane-byplane active cavitation mapping for high-intensity focused ultrasound in free field and pulsatile flow," Ultrasonics, vol. 69, pp. 166-181, Jul. 2016.

[13] P. R. Stepanishen, "Pulsed transmit/receive response of ultrasonic piezoelectric transducers," J. Acoust. Soc. Amer., vol. 69, no. 6 , pp. 1815-1827, 1981.

[14] R. N. Carpenter and P. R. Stepanishen, "An improvement in the range resolution of ultrasonic pulse echo systems by deconvolution," J. Acoust. Soc. Amer., vol. 75, no. 4, pp. 1084-1091, 1984.

[15] J. C. Lacefield and R. C. Waag, "Spatial coherence analysis applied to aberration correction using a two-dimensional array system," J. Acoust. Soc. Amer., vol. 112, no. 6, pp. 2558-2566, Dec. 2002. 
[16] T. D. Mast, "Fresnel approximations for acoustic fields of rectangularly symmetric sources," J. Acoust. Soc. Amer, vol. 121, no. 6, pp. 3311-3322, Jun. 2007.

[17] R. J. Zemp, J. Tavakkoli, and R. S. C. Cobbold, "Modeling of nonlinear ultrasound propagation in tissue from array transducers," J. Acoust. Soc. Amer., vol. 113, no. 1, pp. 139-152, Jan. 2003.

[18] M. Hlawitschka, R. J. McGough, K. W. Ferrara, and D. E. Kruse, "Fast ultrasound beam prediction for linear and regular two-dimensional arrays," IEEE Trans. Ultrason., Ferroelect., Freq. Control, vol. 58, no. 9, pp. 2001-2012, Sep. 2011.

[19] T. D. Mast, "Convolutional modeling of diffraction effects in pulseecho ultrasound imaging," J. Acoust. Soc. Amer, vol. 128, no. 3, pp. EL99-EL104, Sep. 2010.

[20] D. W. Yang, D. Xing, S. H. Yang, and L. Z. Xiang, "Fast full-view photoacoustic imaging by combined scanning with a linear transducer array," Opt. Exp., vol. 15, no. 23, pp. 15566-15575, Nov. 2007.

[21] S. A. Ermilov et al., "Laser optoacoustic imaging system for detection of breast cancer," J. Biomed. Opt., vol. 14, no. 2, pp. 024007-1-024007-14, Mar./Apr. 2009.

[22] M. Á. A. Caballero, A. Rosenthal, A. Buehler, D. Razansky, and V. Ntziachristos, "Optoacoustic determination of spatio-temporal responses of ultrasound sensors," IEEE Trans. Ultrason., Ferroelect., Freq. Control, vol. 60, no. 6, pp. 1234-1244, Jun. 2013.

[23] M. Á. A. Caballero, J. Gateau, X.-L. Déan-Ben, and V. Ntziachristos, "Model-based optoacoustic image reconstruction of large threedimensional tomographic datasets acquired with an array of directional detectors," IEEE Trans. Med. Imag., vol. 33, no. 2, pp. 433-443, Feb. 2014

[24] K. Wang, R. Su, A. A. Oraevsky, and M. A. Anastasio, "Investigation of iterative image reconstruction in three-dimensional optoacoustic tomography," Phys. Med. Biol., vol. 57, no. 17, pp. 5399-5423, Sep. 2012

[25] M. D. Cahill and A. C. Baker, "Numerical simulation of the acoustic field of a phased-array medical ultrasound scanner," J. Acoust. Soc. Amer., vol. 104, no. 3, pp. 1274-1283, Sep. 1998.

[26] D. A. Guenther and W. F. Walker, "A method for accurate in silico modeling of ultrasound transducer arrays," Ultrasonics, vol. 49, nos. 4-5, pp. 404-412, May 2009.

[27] H. Hasegawa and H. Kanai, "Adaptive beamformer incorporating with element directivity," in Proc. IEEE Ultrason. Symp., Taipei, Taiwan, Oct. 2015, pp. 1-4.

[28] N. McDannold, C. D. Arvanitis, N. Vykhodtseva, and M. S. Livingstone, "Temporary disruption of the blood-brain barrier by use of ultrasound and microbubbles: Safety and efficacy evaluation in rhesus macaques," Cancer Res., vol. 72, no. 14, pp. 3652-3663, Jul. 2012.

[29] J. H. Hwang, J. Tu, A. A. Brayman, T. J. Matula, and L. A. Crum, "Correlation between inertial cavitation dose and endothelial cell damage in vivo," Ultrasound Med. Biol., vol. 32, no. 10, pp. 1611-1619, Oct. 2006

[30] C. R. Jensen, R. W. Ritchie, M. Gyöngy, J. R. T. Collin, T. Leslie, and C. C. Coussios, "Spatiotemporal monitoring of high-intensity focused ultrasound therapy with passive acoustic mapping," Radiology, vol. 262, no. 1, pp. 252-261, May 2012.

[31] J. Li, P. Stoica, and Z. Wang, "On robust Capon beamforming and diagonal loading," IEEE Trans. Signal Process., vol. 51, no. 7, pp. 1702-1715, Jul. 2003.

[32] A. D. Pierce, Acoustics: An Introduction to its Physical Principles and Applications. Melville, NY, USA: Acoustical Society America, 1981.

[33] R. J. Bobber, Underwater Electroacoustic Measurements. Washington, DC, USA: Naval Research Laboratory, 1970.

[34] G. R. Harris, "Hydrophone measurements in diagnostic ultrasound fields," IEEE Trans. Ultrason., Ferroelect., Freq. Control, vol. 35, no. 2, pp. 87-101, Mar. 1988.

[35] W. Marczak, "Water as a standard in the measurements of speed of sound in liquids," J. Acoust. Soc. Amer., vol. 102, no. 5, pp. 2776-2779, Nov. 1997.

[36] J. J. Choi and C.-C. Coussios, "Spatiotemporal evolution of cavitation dynamics exhibited by flowing microbubbles during ultrasound exposure," J. Acoust. Soc. Amer., vol. 132, no. 5, pp. 3538-3549, Nov. 2012.

[37] S. Bhatnagar, H. Schiffter, and C.-C. Coussios, "Exploitation of acoustic cavitation-induced microstreaming to enhance molecular transport," J. Pharmaceutical Sci., vol. 103, no. 6, pp. 1903-1912, Jun. 2014.

[38] N. Hockham, C. C. Coussios, and M. Arora, "A real-time controller for sustaining thermally relevant acoustic cavitation during ultrasound therapy," IEEE Trans. Ultrason., Ferroelect., Freq. Control, vol. 57, no. 12, pp. 2685-2694, Dec. 2010.
[39] M. C. Junger and D. Feit, Sound, Structures, and Their Interaction. Melville, NY, USA: Acoustical Society America, 1986.

[40] Vantage Product Specification, Verasonics Inc., Kirkland, WA, USA, Sep. 2015.

[41] K. J. Haworth, V. A. Salgaonkar, N. M. Corregan, C. K. Holland, and T. D. Mast, "Using passive cavitation images to classify high-intensity focused ultrasound lesions," Ultrasound Med. Biol., vol. 41, no. 9, pp. $2420-2434$, Sep. 2015.

[42] J. R. T. Collin and C. C. Coussios, "Quantitative observations of cavitation activity in a viscoelastic medium," J. Acoust. Soc. Amer. vol. 130, no. 5, pp. 3289-3296, Nov. 2011.

[43] K. T. Rich and T. Mast, "Accuracy of a bistatic scattering substitution technique for calibration of focused receivers," J. Acoust. Soc. Amer. vol. 138, no. 5, pp. EL469-EL473, Nov. 2015.

[44] E. Lyka, "Passive acoustic mapping for improved detection and localization of cavitation activity," Ph.D. dissertation, Dept. Eng. Sci., Univ. Oxford, Oxford, U.K., 2017.

[45] E. Lyka, C. Coviello, R. Kozick, and C. C. Coussios, "Sum-of-harmonics method for improved narrowband and broadband signal quantification during passive monitoring of ultrasound therapies," J. Acoust. Soc. Amer., vol. 140, no. 1, pp. 741-754, Jul. 2016.

[46] F. Duck, Physical Properties of Tissue: A Comprehensive Reference Book. San Diego, CA, USA: Academic, 1990.

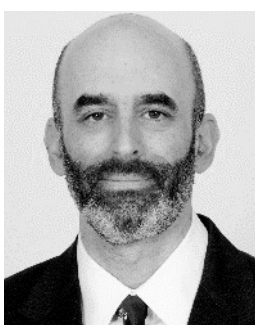

Michael D. Gray received the B.S. degree in mechanical engineering from Tufts University, Medford, MA, USA, in 1990, and the M.S. and $\mathrm{Ph} . \mathrm{D}$. degrees in mechanical engineering from the Georgia Institute of Technology, Atlanta, GA, USA, in 1992 and 2015, respectively.

From 1992 to 2015, he was a Research Engineer at the Georgia Institute of Technology. Since 2015 , he has been a Senior Research Fellow with the Biomedical Ultrasonics, Biotherapy and Biopharmaceuticals Laboratory, Institute of Biomedical Engineering, University of Oxford, Oxford, U.K. His current research interests include the use of sound, magnetism, and light for targeted drug delivery, the clinical translation of cavitation monitoring techniques, and hearing in marine animals.

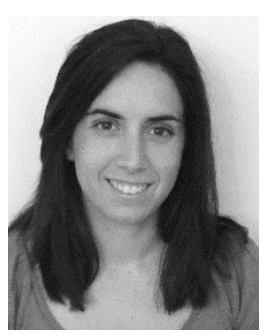

Erasmia Lyka received the M.Eng. degree (Hons.) in electrical and computer engineering from the Aristotle University of Thessaloniki, Thessaloniki, Greece, in 2011, and the M.Sc. and D.Phil. (Ph.D.) degrees in biomedical engineering from the University of Oxford, Oxford, U.K., in 2012 and 2017, respectively. She received the United Kingdom's Engineering and Physical Sciences Research Council Departmental Studentship for the Ph.D. degree.

She is currently a Post-Doctoral Research Assistant of therapeutic ultrasound in the Biomedical Ultrasonics, Biotherapy and Biopharmaceuticals Laboratory, Institute of Biomedical Engineering, University of Oxford. Her current research interests include therapeutic ultrasound and array beamforming.

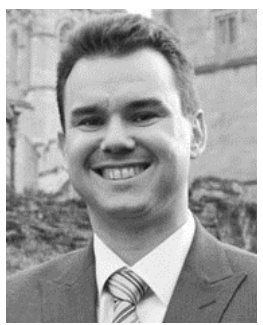

Constantin C. Coussios received the B.A., M.Eng., M.A., and Ph.D. degrees from the University of Cambridge, Cambridge, U.K., in 1997, 1998, 2000, and 2002, respectively.

$\mathrm{He}$ is currently the Director of the Oxford Institute of Biomedical Engineering, a Statutory Professor of Biomedical Engineering, and the Head of the Biomedical Ultrasonics, Biotherapy and Biopharmaceuticals Laboratory, University of Oxford, Oxford, U.K. He is also the Co-Founder and the Director of two biomedical technology companies: OrganOx Ltd., Oxford, and OxSonics Ltd., Oxford.

Prof. Coussios was a recipient of the F.V. Hunt Postdoctoral Fellowship of the Acoustical Society of America (ASA) from 2002 to 2003, the International Society in Therapeutic Ultrasound (ISTU) Frederic Lizzi Award in 2012, and the ASA's 2012 Bruce Lindsay Award. He received the Silver Medal by the UK's Royal Academy of Engineering in 2017. He was elected a fellow of the ASA in 2009. He served as the Secretary General for the International Society in ISTU from 2006 to 2010 . 